\title{
Interlayer Debonding Inspection in Airport Pavements by MIMO GPR System
}

\author{
Lilong Zou (1), Fabio Tosti (1), Amir M. Alani (1), Motoyuki Sato (2) \\ (1) University of West London (UWL), School of Computing and Engineering, London, United Kingdom \\ (Lilong.Zou@uwl.ac.uk; Fabio.Tosti@uwl.ac.uk; Amir.Alani@uwl.ac.uk), (2) Tohoku University, Center for Northeast \\ Asian Studies, Sendai, Japan (Motoyuki.Sato.b3@tohoku.ac.jp)
}

The integrity and flatness of airport pavement facilities are important to maintain safe operations of aircrafts. Even a small defect and resulting debris can cause catastrophic accidents and, therefore, anomalies must be accurately detected for the first time before major damage occurs. To this effect, it is necessary to develop a low-cost, efficient, and accurate inspection technology to detect the anomalies in airport concrete pavements. In recent years, non-destructive testing (NDT) methods have been widely used in airport pavement inspection and maintenance due to the provision of reliable and efficient information. Amongst the NDT techniques, GPR can provide optimal resolutions for different applications in civil engineering due to the ultra-wide frequency band configuration [1][2]. However, for the investigation of airport pavement facilities main challenges are how to extract information from the reflections by small anomalies [3][4].

In this research, we used a MIMO GPR system to inspect the interlayer debonding in a large area of an airport pavement. A special set of antenna arrangements of the system can obtain common mid-point (CMP) gathers during a common offset survey simultaneously. The existence of interlayer debonding affects the phase of the reflection signals, and the phase disturbance can be quantified by wavelet transform. Therefore, an advanced approach that uses the average entropy of the wavelet transform parameters in a CMP gathers to detect the interlayer debonding in airport pavements is proposed.

The aim of this research is to provide more significant and accurate information for airport pavement inspections using a MIMO GPR system. To this extent, the wavelet entropy analysis is applied to identify the interlayer debonding existed in the shallow region. The proposed approach was then evaluated by field tests on an airport taxiway. The results were validated by on-site coring and demonstrate that the regions with high entropy correspond to the regions where tiny voids occurred. The proposed method has proven potential to detect the interlayer debonding of the pavement model accurately and efficiently.

\section{References}

[1] Alani, A. M. et al., 2020. Reverse-Time Migration for Evaluating the Internal Structure of Tree-Trunks Using Ground-Penetrating Radar. NDT\&E International, vol.115, pp:102294.

[2] Zou, L. et al., 2020. Mapping and Assessment of Tree Roots using Ground Penetrating Radar with LowCost GPS. Remote Sensing, vol.12, no.8, pp:1300.

[3] Zou, L. et al., 2020. On the Use of Lateral Wave for the Interlayer Debonding Detecting in an Asphalt Airport Pavement Using a Multistatic GPR System. IEEE Transaction on Geoscience and Remote Sensing, vol. 58, no. 6, pp. 4215-4224.

[4] Zou, L. et al., 2021. Study on Wavelet Entropy for Airport Pavement Debonded Layer Inspection by using a Multi-Static GPR System. Geophysics, in press. 